\title{
The etiology, clinical profile, and outcome of diffuse alveolar hemorrhage in children: a ten-year single-center experience
}

\author{
Yuanyuan Qi, Libo Wang, Liling Qian, Xiaobo Zhang \\ Department of Respiratory Disease, Children's Hospital of Fudan University, National Children's Medical Center, Shanghai, China \\ Contributions: (I) Conception and design: Y Qi; (II) Administrative support: X Zhang; (III) Provision of study materials or patients: L Wang; (IV) \\ Collection and assembly of data: Y Qi; (V) Data analysis and interpretation: Y Qi; (VI) Manuscript writing: All authors; (VII) Final approval of \\ manuscript: All authors. \\ Correspondence to: Yuanyuan Qi. No. 399 Wanyuan Road, Shanghai, 201102, China. Email: qiyuanyuan3636@hotmail.com.
}

\begin{abstract}
Background: Diffuse alveolar hemorrhage (DAH) is a life-threatening syndrome that may be caused by numerous disorders. There is scant data on the etiology and characteristics of DAH in children.

Methods: We retrospectively reviewed the clinical records of patients admitted to a tertiary pediatric hospital with DAH over a 10 -year period. The syndrome was classified into five groups according to different etiologies, and the characteristics and outcomes of patients were compared.

Results: A total of 74 children were included in the study. Idiopathic pulmonary hemosiderosis (IPH) was the most frequent cause (64.9\%), followed by miscellaneous causes (infection and other conditions) (16.2\%), immune-mediated disorders (9.5\%), liver dysfunction (5.4\%), and cardiovascular disorders (4.1\%). The median age of the patients was 3.5 years (ranging from 1.5 to 7 years), and no difference was found in the proportion of fever, crackles, and pulmonary infiltrates among the five etiological groups. There was no difference in the proportion of blood transfusions among the groups. Cardiac catheterization was performed on 31 patients for whom the diagnostic workup was negative and were suspected of having IPH, and abnormal signs were observed and bronchial artery embolization (BAE) was performed in all those patients. The patients with IPH had the lowest mortality, while those with DAH secondary to liver failure had the highest mortality. Patients in BAE group had a shorter duration of corticosteroids and a lower relapse rate than non-BAE group.

Conclusions: Idiopathic pulmonary hemosiderosis is a common etiology of DAH and has a good prognosis in children. Vascular abnormalities were observed in the patients with IPH. Further studies are needed to clarify the role of vascular malformations in IPH.
\end{abstract}

Keywords: Diffuse alveolar hemorrhage; idiopathic pulmonary hemosiderosis; etiology; bronchial artery embolization

Submitted Jun 18, 2021. Accepted for publication Sep 13, 2021.

doi: $10.21037 / \mathrm{tp}-21-283$

View this article at: https://dx.doi.org/10.21037/tp-21-283

\section{Introduction}

Diffuse alveolar hemorrhage (DAH) is a life-threatening condition; the name refers to hemorrhage originating from the pulmonary microvasculature (1). Clinical presentation of DAH includes hemoptysis, anemia, hypoxemic respiratory distress, and diffuse radiographic pulmonary infiltrates. Although DAH is a rare disease, it may cause significant morbidity. Early recognition is essential for appropriate treatment and the prevention of lethal events.

A spectrum of disorders is associated with DAH (2). A study based on biopsy specimens from 34 cases of DAH showed that nearly one-third of the cases were caused by Wegener's granulomatosis (3). de Prost et al. reviewed the etiology and prognosis of a series of 112 immunocompetent patients (4). There may be a difference in the etiology and prognosis of DAH between children and adults. However, 
little is known about the etiology and clinical features of DAH in children.

We investigated a series of pediatric patients with DAH in a single center over ten years, aiming to describe the causes of DAH and their relative frequency and to compare the clinical features and prognosis according to etiology. We present the following article in accordance with the STROBE reporting checklist (available at https://dx.doi. org/10.21037/tp-21-283).

\section{Methods}

\section{Study population}

This retrospective study was performed at a tertiarycare children's hospital, the Children's Hospital of Fudan University, China. We analyzed the medical records of patients with a diagnosis of DAH documented at any time during admission to the hospital from 1 July, 2010, to 30 June, 2020. For patients with several admissions for DAH of the same etiology, only the first admission was considered for etiology analysis.

The diagnosis of DAH was based on a combination of clinical presentation of hemoptysis, respiratory difficulty, and evidence of bilateral infiltrate on computed tomography (CT) of the chest, and was confirmed by flexible bronchoscopy with bronchoalveolar lavage or hemorrhage from an endotracheal tube.

A detailed record review was performed to determine the diagnoses associated with pulmonary hemorrhage in this cohort. Patients with congenital heart disease, primary pulmonary hypertension, known trauma, foreign bodies, and pulmonary hemorrhage during cardiopulmonary resuscitation were excluded from the study. Preterm infants who developed hemorrhage prior to 38 weeks of corrected gestational age were also excluded.

\section{Data collection}

Data collected from the electronic medical records included demographics, symptoms, comorbid conditions, laboratory tests, and radiographic and angiographic data, as well as interventions, hospital length of stay and in-hospital mortality. For idiopathic pulmonary hemosiderosis (IPH) patients, follow-up data from the outpatient clinic was also collected, including the duration of corticosteroids use and frequency of relapses. The study was conducted in accordance with the Declaration of Helsinki (as revised in 2013). The study was approved by the Medical Ethics Committee of the Children's Hospital of Fudan University (Shanghai, China; No. 2018-287) and individual consent for this retrospective analysis was waived.

The blood tests in our center included routine and immunological/ rheumatologic bloodwork, and coagulation function. Flexible bronchoscopy was performed to exclude local bleeding and collect bronchoalveolar lavage. Chest radiography, CT, and echocardiography were initially performed. Since 2016, angiography was performed in all patients from whom informed consent was obtained after their workup failed to reveal a specific hemoptysis etiology. No patients underwent lung biopsy in the present study.

\section{Definitions and classification of the underlying causes of $D A H$}

The patients were divided into five etiological groups: immune-mediated disorders, IPH, liver dysfunction, cardiovascular causes, and miscellaneous causes (infection and others).

Cardiovascular diseases were diagnosed when abnormal conditions were found by echocardiography or heart catheterization. The diagnosis of IPH was determined when all relevant investigations for a possible etiology were negative. The diagnosis of infection was established by positive microbiological or serological tests, or established by a specialist according to clinical findings and high levels of C-reactive protein or procalcitonin. Liver dysfunction refers to acute and chronic liver failure. Immune-mediated disorders included systemic vasculitis, connective tissue diseases, antiglomerular basement membrane antibody disease, and Evans syndrome.

\section{Statistical analysis}

All data was processed using the SPSS software. The parametric continuous variables were reported as mean \pm standard deviation, and nonparametric continuous variables were reported as the median and interquartile range. Categorical variables were reported as percentages. The differences among etiological groups were analyzed using one-way analysis of variance (ANOVA) or Kruskal-Wallis $\mathrm{H}$ test for continuous variables and Fisher's exact test for categorical variables. For those analyses in which the overall test was significant, a post-hoc test of Bonferroni or pairwise comparisons controlling for Type I error across tests with the Bonferroni adjustment was performed for continuous 
Table 1 Underlying etiology of DAH

\begin{tabular}{|c|c|}
\hline Etiology & Number (\%) \\
\hline Miscellaneous causes & $12(16.2)$ \\
\hline Sepsis & 6 \\
\hline Pneumonia-induced acute respiratory distress syndrome (ARDS) & 3 \\
\hline Influenza B infection & 1 \\
\hline Drowning & 1 \\
\hline Immune-mediated disorders & $7(9.5)$ \\
\hline Anti-neutrophil cytoplasmic antibody (ANCA) associated granulomatous vasculitis & 3 \\
\hline Liver dysfunction & $4(5.4)$ \\
\hline Acute liver failure & 3 \\
\hline Favism & 1 \\
\hline Cardiovascular disorders & $3(4.1)$ \\
\hline Heart failure & 2 \\
\hline Vascular malformation & 1 \\
\hline
\end{tabular}

variables, and Fisher's exact test with Bonferroni corrected $\mathrm{P}$ values used for statistical significance was performed for categorical variables. The differences between two groups were analyzed using Student's $t$ test or the Mann-Whitney $\mathrm{U}$ test for continuous variables and chi-square test for categorical variables. Statistical significance was set at $\mathrm{P}<0.05$.

\section{Results}

\section{Etiology of DAH}

A total of 74 patients were included in this study. The causes of DAH were classified into five etiological groups (Table 1). Possible IPH was the most frequent cause (48 cases, 64.9\%). Immune-mediated disorders were diagnosed in 7 patients: three patients with anti-neutrophil cytoplasmic antibody (ANCA)-related vasculitis, three patients with Goodpasture syndrome, and one patient with Evans syndrome (9.5\%). Liver dysfunction was found in four patients: three patients with acute liver function failure and one patient with Favism (5.4\%). Cardiovascular diseases were found in 3 patients: two patients with heart failure and one patient with vascular malformation (4.1\%).

In the miscellaneous causes group (12 cases, 16.2\%), the most common etiology was infection, which included pneumonia-induced acute respiratory distress syndrome (ARDS) (3 cases), sepsis (6 cases), and virus infection (2 cases). The infection group included four immunosuppressed patients (one with primary immunodeficiency; one with primary immunodeficiency who had received a stem cell transplant; one with Langerhans cell histiocytosis, who was receiving chemotherapy; and one with leukemia, who had received a bone marrow transplant). All immunocompromised patients were diagnosed with sepsis. Of the seven immunocompetent patients (aside from the drowning patient), one was diagnosed with Enterovirus A71 (EV71) infection and one was diagnosed with influenza $B$ virus infection.

\section{Patient characteristics with different etiologies}

Demographic characteristics are shown in Table 2. The median age was 3.5 years (ranging from 1.5 to 7 years). The median age of the IPH group was lower than that of the immune mediated group and higher than those of the liver 
Table 2 Patient characteristics and outcome according to the etiology

\begin{tabular}{|c|c|c|c|c|c|c|c|}
\hline Variables & $\begin{array}{l}\text { All patients } \\
\qquad(\mathrm{n}=74)\end{array}$ & $\begin{array}{l}\text { Immune related } \\
\qquad(\mathrm{n}=7)\end{array}$ & $\begin{array}{l}\text { Liver dysfunction } \\
\qquad(\mathrm{n}=4)\end{array}$ & $\begin{array}{l}\text { Cardiovascular } \\
\text { causes }(n=3)\end{array}$ & $\begin{array}{l}\text { Miscellaneous } \\
\text { (infection and } \\
\text { other conditions) } \\
\quad(n=12)\end{array}$ & $\begin{array}{c}\mathrm{IPH} \\
(\mathrm{n}=48)\end{array}$ & $P$ value \\
\hline Age, year & $3.5(1.5-7)$ & $9(6.0-11.0)$ & $0.15(0.1-0.425)$ & $13(0.6-13)$ & $1.5(0.7-5.5)$ & $4.0(2-6.8)$ & 0.001 \\
\hline Male gender, \% & $38(51.4)$ & $4(57.1)$ & $4(100.0)$ & $3(100.0)$ & 4 (33.3) & $23(47.9)$ & 0.078 \\
\hline \multicolumn{8}{|l|}{ Presenting symptoms } \\
\hline Fever & $29(39.2)$ & $2(28.6)$ & $2(50.0)$ & $1(33.3)$ & $8(66.7)$ & $16(33.3)$ & 0.288 \\
\hline Cough & $45(60.8)$ & $3(42.9)$ & $0(0)$ & $1(33.3)$ & $3(25.0)$ & 38 (79.2) & $<0.001$ \\
\hline Anemia & $62(83.8)$ & $7(100.0)$ & $3(75.0)$ & $0(0)$ & 7 (58.3) & 45 (93.8) & $<0.001$ \\
\hline Hypoxia & $32(43.2)$ & $4(57.1)$ & $4(100.0)$ & $2(66.7)$ & $11(91.7)$ & $11(22.9)$ & $<0.001$ \\
\hline Clubbing & $2(2.7)$ & $0(0)$ & $0(0)$ & $1(33.3)$ & $0(0)$ & $1(2.1)$ & 0.024 \\
\hline Crackles & $20(27.0)$ & $0(0)$ & $0(0)$ & $1(33.3)$ & $6(50.0)$ & $13(27.1)$ & 0.123 \\
\hline $\mathrm{Hb}, \mathrm{g} / \mathrm{L}$ & $76.8 \pm 32$ & $67.3 \pm 22.6$ & $82.8 \pm 15.2$ & $159 \pm 64.9$ & $94.4 \pm 30.1$ & $68.1 \pm 22.6$ & 0.002 \\
\hline Pulmonary infiltrates & $66(89.2)$ & $7(100.0)$ & $3(75.0)$ & $3(100.0)$ & $9(75.0)$ & $44(91.7)$ & 0.309 \\
\hline DAH classical triad ${ }^{\star}$ & $36(48.6)$ & $4(57.1)$ & $1(25.0)$ & $0(0)$ & 4 (33.3) & $27(56.3)$ & 0.192 \\
\hline
\end{tabular}

${ }^{*}$, refers to hemoptysis, anemia and pulmonary infiltrates on chest imaging.

dysfunction and miscellaneous causes groups. There were no significant gender differences among the groups. The mean hemoglobin level was $76.8 \pm 32 \mathrm{~g} / \mathrm{L}$. The hemoglobin level was the highest in the cardiovascular disease group, and there were no significant differences among immunemediated, liver dysfunction, and IPH groups.

The DAH triad of hemoptysis, iron deficiency anemia, and pulmonary infiltrates was observed in $48.6 \%$ of the patients. Ten patients in the IPH group and three patients in the immune-mediated group did not have hemoptysis. No difference was observed in the proportion of fever, hemoptysis, crackles, and pulmonary infiltrates between the IPH group and the other groups. However, in the liver dysfunction group, none of the patients had cough, and only one patient had hemoptysis. None of the patients in the cardiovascular disease group had anemia.

The proportion of dyspnea and hypoxia was lowest in the IPH group, followed by the immune-mediated group. Patients with miscellaneous causes (infection and drowning) had the highest proportions of dyspnea $(91.7 \%)$ and hypoxia (91.7\%).

\section{Chest radiography and angiography}

All patients underwent chest radiography or CT scans. Chest CT or helical chest CT angiograms with intravenous contrast on these patients showed no abnormal bronchial vessels. Chest CT showed nonspecific bilateral ground glass opacities, multiple patchy shadows, diffuse alveolar infiltrates, grid shadows, diffuse cystic shadows, and interstitial changes. Fifty-three patients underwent bronchoscopy, and no local bleeding was observed.

Cardiac catheterization was performed on 31 patients whose diagnostic workup was negative and who were thus suspected of having IPH. It is noteworthy that all the patients who were suspected of IPH and underwent angiography had abnormal signs, including bilateral abnormal signs in 19 cases and unilateral abnormal signs in 12 cases. These abnormalities included bronchial artery pulmonary-artery fistula $(\mathrm{n}=8,25.8 \%)$, bronchial arterypulmonary vein fistula $(\mathrm{n}=3,9.7 \%)$, enlarged or tortuous vessels $(n=25,80.6 \%)$, hypervascularity $(n=24,77.4 \%)$, and leakage of contrast medium $(n=3,9.7 \%)$. All the patients underwent bronchial artery embolization (BAE) (Table S1). 
Table 3 Treatment, hospital duration and outcome

\begin{tabular}{|c|c|c|c|c|c|c|c|}
\hline Variables & $\begin{array}{l}\text { All patients } \\
\quad(n=74)\end{array}$ & $\begin{array}{l}\text { Immune-mediated } \\
\qquad(\mathrm{n}=7)\end{array}$ & $\begin{array}{c}\text { Liver } \\
\text { dysfunction } \\
(n=4)\end{array}$ & $\begin{array}{l}\text { Cardiovascular } \\
\text { causes }(n=3)\end{array}$ & $\begin{array}{c}\text { Miscellaneous } \\
\text { (infection and } \\
\text { other conditions) } \\
\quad(n=12)\end{array}$ & $\begin{array}{c}\mathrm{IPH} \\
(\mathrm{n}=48)\end{array}$ & $P$ value \\
\hline Blood transfusion & $53(71.6)$ & $6(85.7)$ & $4(100.0)$ & $1(33.3)$ & 7 (58.3) & $35(72.9)$ & 0.280 \\
\hline IVIG & $23(31.1)$ & $4(66.7)$ & $2(50.0)$ & $1(33.3)$ & $6(50.0)$ & 9 (18.8) & 0.013 \\
\hline Mechanical ventilation & $25(33.8)$ & $4(66.7)$ & $3(60.0)$ & $2(66.7)$ & $10(83.3)$ & $6(12.5)$ & $<0.001$ \\
\hline Hospital length of stay, days & $13 \pm 1.5$ & $14.3 \pm 12.7$ & $3.8 \pm 2.2$ & $12.3 \pm 2.5$ & $19.7 \pm 21.7$ & $11.9 \pm 9.7$ & 0.145 \\
\hline In hospital mortality & $19(25.7)$ & $3(42.9)$ & $4(100.0)$ & $2(66.7)$ & $9(75.0)$ & $1(2.1)$ & $<0.001$ \\
\hline
\end{tabular}

Table 4 Demographic characteristics and prognosis in BAE and non-BAE patients

\begin{tabular}{lccc}
\hline Variables & BAE $(n=31)$ & Non-BAE $(n=17)$ & $P$ value \\
\hline Age at diagnosis, years & $5.2 \pm 4.0$ & $4.0 \pm 3.2$ & 0.311 \\
Male gender, \% & $11(35.5)$ & $12(70.6)$ & 0.034 \\
Disease course, months & $20.1 \pm 31.2$ & $17.7 \pm 37.1$ & 0.812 \\
$\mathrm{Hb}>120 \mathrm{~g} / \mathrm{L}, \%$ & $21(67.7)$ & $14(82.4)$ & 0.330 \\
Duration of corticosteroids, months & $5.5[3-10]$ & $10[8-15]$ & 0.020 \\
Relapse, \% & $11(35.5)$ & $12(70.6)$ & 0.021 \\
Frequency of relapses & $0[0-1]$ & $2[0-2]$ & $<0.001$ \\
\hline
\end{tabular}

\section{Treatment and prognosis}

There was no difference among the groups in the percentage of patients who received blood transfusions. The IPH group had a lower percentage of intravenous immunoglobulin (IVIG) therapy, oxygen treatment, and mechanical ventilation (Table 3). This group had the lowest mortality, while the liver dysfunction group had the highest mortality.

\section{Patient characteristics and prognosis for IPH}

In total, there were 48 patients with IPH. One patient was positive for antinuclear antibodies, three patients were weakly positive for perinuclear anti-neutrophil cytoplasmic antibodies, one patient was weakly positive for antiextractable nuclear antigen, and one patient was weakly positive for anti-mitochondrial antibody. Moreover, eight patients were positive for specific cow's milk IgE. No patient with Down syndrome was found.

All patients with IPH received prednisolone or methylprednisolone. Thirty-one patients received BAE therapy. Three of them had received azathioprine, leflunomide or hydroxychloroquine before BAE therapy.

The patients with IPH were classified into BAE and non-BAE groups. There were no differences in the age and disease course between BAE and non-BAE groups. The ratio of female was higher in the BAE group than in the non-BAE group. We analyzed the outcome data of 47 patients within 18 months after discharge. The median duration of corticosteroids was lower in BAE group than in non-BAE group. Moreover, there was a lower relapse rate in BAE group (Table 4). Three patients who experienced relapses underwent repeat BAE in the BAE group.

\section{Discussion}

Diffuse alveolar hemorrhage is a syndrome with various presentations that can complicate many clinical conditions. In this study, we described the causes of DAH and their relative frequency in a series of pediatric patients.

The most common underlying etiology was IPH, 
accounting for $65 \%$ of all patients. The proportion of immune-mediated disorders was relatively low in this study, which is consistent with previous studies in children (5). In the immune-mediated group, the proportion of Goodpasture syndrome was higher than that reported in adults (6-8). These results demonstrated that the etiology of DAH in children is very different from its etiology in adults, and the differences may be due to unique disease characteristics in children (9).

Infection is also a major cause of DAH that should be considered in the diagnostic workup. In this study, infectious etiology was diagnosed in four immunocompromised patients and seven immunocompetent patients. Consistent with previous reports, DAH is common in patients who received hematopoietic stem cell transplantation. The most commonly reported pathogens of DAH in patients with normal immune function are influenza A virus, dengue fever, and Staphylococcus aureus, whereas the frequently reported pathogens of DAH in immunocompromised patients are cytomegaloviruses, adenoviruses, invasive fungal infections, Mycoplasma pneumonia, and Legionella (9). In this study, no specific pathogen was detected in immunocompromised patients, EV71 and influenza B were detected in two immunocompetent patients. No specific bacteria were found among the other cases of infectious DAH. This may be due to a higher rate of use of antibiotics.

Four patients in this study with liver function failure were diagnosed with DAH. DAH caused by liver failure has rarely been reported in adults, a previous literature reported pulmonary hemorrhage in a pediatric patient with liver failure (10). A possible mechanism may be coagulation dysfunction secondary to liver function.

In this study, 31 patients suspected of IPH underwent angiography. Remarkably, abnormal angiographic signs were observed in all these patients. These abnormalities could not be detected in chest enhancement CT. This finding indicates that vascular abnormalities are very common in patients with IPH. As an exclusive diagnosis, the pathogenesis of IPH remains unclear. Recent studies have focused on the role of vascular abnormalities in the pathogenesis of IPH. Alimi et al. documented that Down syndrome patients had a higher risk of developing IPH $(11,12)$, and children with Down syndrome had more pulmonary malformations and intrapulmonary bronchopulmonary anastomoses (13). Moreover, in the present study, we found that BAE patients had a shorter corticosteroids duration and a lower relapse rate than Non-BAE patients. Collectively, these results indicate that vascular malformation is related to IPH, but whether the vascular abnormalities are a cause of IPH, a secondary change of IPH, or a simultaneous problem, has not been identified. There is a possibility that, although there were abnormal signs in catheterization, it did not cause alveolar bleeding, or the abnormal signs may lead to an increase in capillary pressure and susceptibility to bleeding. These results highlight the importance of awareness of the potential mechanism for IPH.

Cardiac catheterization is mainly used to identify and treat massive hemoptysis and recurrent hemoptysis from the bronchial circulation (14-16). It is not routinely performed in patients without obvious vascular abnormalities in chest CT images (17). Remarkably, abnormal signs were observed in cardiac catheterization for many children diagnosed with IPH in the present study. Moreover, patients with BAE showed a better prognosis than the subjects without BAE. Although the role of abnormal signs in IPH remains unclear, there is no doubt that these findings are of great significance and suggest that angiography should be performed in children with DAH of unknown cause.

An autoimmune etiology for IPH has been previously hypothesized by some investigators. In the present study, only six patients with weakly positive auto-immune antibodies were observed, which might be associated with infections or other factors. However, the auto-immune status in these patients need further follow up.

The reported mortality rate for DAH is high in adults, ranging from $20 \%$ to $50 \%(4,18,19)$. However, the outcome of DAH varies depending on the cause. Survival trends to increase in immune DAH, and the reported mortality was about $30 \%$ in a recent series of patients $(20,21)$. Little is known about the prognosis for children with a specific etiology. The mortality rate in this study was $33.3 \%$, which is consistent with the data reported in adults. In this study, patients with infection and liver failure had a high mortality rate. The DAH syndrome has been reported mostly in H1N1 virus infection and is associated with high mortality $(9,22-24)$. In this study, we reported one patient with influenza B and one patient with EV71, and both of them died during their hospital stays. This study also documented that viral infection with DAH had a high mortality rate, and early identification and treatment are critical.

The present study had several limitations. First, because lung biopsy is an invasive method with high morbidity and mortality for children, it is not recommended if the diagnostic work-up strongly suggests DAH. No patients in this study underwent lung biopsy. However, lung biopsy is 
needed when treatment fails. Second, this was a retrospective study. Angiography and BAE were performed after 2016 instead of being randomly allocated. Therefore, there may have been a selection bias. Moreover, the role of abnormal angiographic signs has not been well studied, and it is not possible to associate the angiographic signs conclusively with the pathogenesis of IPH. Prospective randomized controlled studies and long-term follow-up are needed to elucidate the impact of vascular abnormalities on IPH.

In conclusion, this study describes the etiology, clinical features and prognosis of DAH in children. The most common cause of DAH in children is IPH. Bronchial artery catheterization should be considered as a routine examination for DAH without a specific etiology. Further investigation is required to elucidate the relationship between vascular malformations and IPH.

\section{Acknowledgments}

We acknowledge all patients and their parents involved in the study.

Funding: None.

\section{Footnote}

Reporting Checklist: The authors have completed the STROBE reporting checklist. Available at https://dx.doi. org/10.21037/tp-21-283

Data Sharing Statement: Available at https://dx.doi. org/10.21037/tp-21-283

Conflicts of Interest: All authors have completed the ICMJE uniform disclosure form (available at https://dx.doi. org/10.21037/tp-21-283). The authors have no conflicts of interest to declare.

Ethical Statement: The authors are accountable for all aspects of the work in ensuring that questions related to the accuracy or integrity of any part of the work are appropriately investigated and resolved. The study was conducted in accordance with the Declaration of Helsinki (as revised in 2013). The study was approved by the Medical Ethics Committee of the Children's Hospital of Fudan University (Shanghai, China; NO. 2018-287) and individual consent for this retrospective analysis was waived.

Open Access Statement: This is an Open Access article distributed in accordance with the Creative Commons Attribution-NonCommercial-NoDerivs 4.0 International License (CC BY-NC-ND 4.0), which permits the noncommercial replication and distribution of the article with the strict proviso that no changes or edits are made and the original work is properly cited (including links to both the formal publication through the relevant DOI and the license). See: https://creativecommons.org/licenses/by-nc-nd/4.0/.

\section{References}

1. Lara AR, Schwarz MI. Diffuse alveolar hemorrhage. Chest 2010;137:1164-71.

2. Susarla SC, Fan LL. Diffuse alveolar hemorrhage syndromes in children. Curr Opin Pediatr 2007;19:314-20.

3. Travis WD, Colby TV, Lombard C, et al. A clinicopathologic study of 34 cases of diffuse pulmonary hemorrhage with lung biopsy confirmation. Am J Surg Pathol 1990;14:1112-25.

4. de Prost N, Parrot A, Cuquemelle E, et al. Diffuse alveolar hemorrhage in immunocompetent patients: etiologies and prognosis revisited. Respir Med 2012;106:1021-32.

5. de Silva C, Mukherjee A, Jat KR, et al. Pulmonary Hemorrhage in Children: Etiology, Clinical Profile and Outcome. Indian J Pediatr 2019;86:7-11.

6. Alexandre AT, Vale A, Gomes T. Diffuse alveolar hemorrhage: how relevant is etiology? Sarcoidosis Vasc Diffuse Lung Dis 2019;36:47-52.

7. Mirouse A, Parrot A, Audigier V, et al. Severe diffuse alveolar hemorrhage related to autoimmune disease: a multicenter study. Crit Care 2020;24:231.

8. Escuissato DL, Warszawiak D, Marchiori E. Differential diagnosis of diffuse alveolar haemorrhage in immunocompromised patients. Curr Opin Infect Dis 2015;28:337-42.

9. von Ranke FM, Zanetti G, Hochhegger B, et al. Infectious diseases causing diffuse alveolar hemorrhage in immunocompetent patients: a state-of-the-art review. Lung 2013;191:9-18.

10. Son SK, Oh SH, Kim KM, et al. Successful liver transplantation following veno-arterial extracorporeal membrane oxygenation in a child with fulminant Wilson disease and severe pulmonary hemorrhage: a case report. Pediatr Transplant 2012;16:E281-5.

11. Alimi A, Taytard J, Abou Taam R, et al. Pulmonary hemosiderosis in children with Down syndrome: a national experience. Orphanet J Rare Dis 2018;13:60.

12. Taytard J, Nathan N, de Blic J, et al. New insights into 
pediatric idiopathic pulmonary hemosiderosis: the French RespiRare(®) cohort. Orphanet J Rare Dis 2013;8:161.

13. Bush D, Abman SH, Galambos C. Prominent Intrapulmonary Bronchopulmonary Anastomoses and Abnormal Lung Development in Infants and Children with Down Syndrome. J Pediatr 2017;180:156-162.e1.

14. Mal H, Rullon I, Mellot F, et al. Immediate and long-term results of bronchial artery embolization for life-threatening hemoptysis. Chest 1999;115:996-1001.

15. Cordovilla R, Bollo de Miguel E, Nuñez Ares A, et al. Diagnosis and Treatment of Hemoptysis. Arch Bronconeumol 2016;52:368-77.

16. Larici AR, Franchi P, Occhipinti M, et al. Diagnosis and management of hemoptysis. Diagn Interv Radiol 2014;20:299-309.

17. Zaidi SJ, Schweig L, Patel D, et al. A novel approach to the diagnosis and treatment of hemoptysis in infants: A case series. Pediatr Pulmonol 2018;53:1504-9.

18. Gallagher H, Kwan JT, Jayne DR. Pulmonary renal syndrome: a 4-year, single-center experience. Am J Kidney Dis 2002;39:42-7.

19. Lin $Y$, Zheng W, Tian X, et al. Antineutrophil cytoplasmic antibody-associated vasculitis complicated with diffuse alveolar hemorrhage: a study of 12 cases. J Clin Rheumatol 2009;15:341-4.

20. Ednalino C, Yip J, Carsons SE. Systematic Review of Diffuse Alveolar Hemorrhage in Systemic Lupus Erythematosus: Focus on Outcome and Therapy. J Clin Rheumatol 2015;21:305-10.

21. Quintana JH, Aragón CC, Santos VA, et al. Diffuse Alveolar Hemorrhage: A Cohort of Patients With Systemic Lupus Erythematosus. J Clin Rheumatol 2020;26:S153-S157.

22. Gilbert CR, Vipul K, Baram M. Novel H1N1 influenza A viral infection complicated by alveolar hemorrhage. Respir Care 2010;55:623-5.

23. Nakajima N, Sato Y, Katano H, et al. Histopathological and immunohistochemical findings of 20 autopsy cases with 2009 H1N1 virus infection. Mod Pathol 2012;25:1-13.

24. Toolsie O, Tehreem A, Diaz-Fuentes G. Influenza A Pneumonia Associated with Diffuse Alveolar Hemorrhage. A Case Report and Literature Review. Am J Case Rep 2019;20:592-6.
Cite this article as: Qi Y, Wang L, Qian L, Zhang X. The etiology, clinical profile, and outcome of diffuse alveolar hemorrhage in children: a ten-year single-center experience. Transl Pediatr 2021;10(11):2921-2928. doi: 10.21037/tp-21-283 
Supplementary

Table S1 Patient characteristics, angiographic findings, and prognosis of bronchial artery embolization (BAE) $(n=31)$

\begin{tabular}{lc}
\hline Variable & Value \\
\hline Age at diagnosis, years & $5.2 \pm 4.0$ \\
Male gender, \% & $11(35.5)$ \\
Disease Course, months & $20.1 \pm 31.2$ \\
Angiographic findings & \\
Bronchial artery pulmonary-artery fistula, \% & $8(25.8)$ \\
Bronchial artery-pulmonary vein fistula, \% & $3(9.7)$ \\
Enlarged or tortuous vessels, \% & $25(80.6)$ \\
Hypervascularity, \% & $24(77.4)$ \\
Leakage of contrast medium, \% & $3(9.7)$ \\
BAE & \\
Particles, \% & $29(93.5)$ \\
Coils, \% & $2(6.5)$ \\
Remission with 30 days, \% & $31(100.0)$ \\
Relapse during follow-up, \% & $11(35.5)$ \\
Repeat embolization, \% & $3(9.7)$ \\
\hline
\end{tabular}

